\title{
Actividad física, callidad de vida e dolor de hombro en lesión medular
}

João Guerreiro', Pedro Sáenz - López Buñuel2, Sandra Pais', José Tierra Orta ${ }^{2}$

\section{'Departamento de Ortoprotesia}

Escuela Superior de Salud de la Universidad del Algarve

\section{Departamento de Educación Física, Música e Artes Plásticas Facultad de Educación - Universidad de Huelva}

\section{Email: jmguerreiro@ualg.pt}

\section{RESUMEN:}

El objetivo de este estudio es de realizar una revisión bibliográfica sobre la relación entre calidad de vida, prevalencia de dolor de hombro y nivel de actividad física en personas con lesión medular. La lesión medular resulta de una enfermedad, tumor o trauma (Maestro, 2010) donde la calidad de vida es un factor bastante importante y que influye bastante en las actividades (Middleton, Tran, y Craig, 2007). Una diferencia entre dedicación y valorización de trabajo indica menores valores de calidad de vida, menor satisfacción con la salud, actividades de vida diaria, relacionamientos y condiciones de vida (Fekete, Wahrendorf, Reinhardt, Post, y Siegrist, 2014). Además, el uso de silla de ruedas manual en sujetos con lesión medular requiere una carga continua sobre la extremidad superior (S van Drongelen, van der Woude, y Veeger, 2011 ; Stefan Van Drongelen et al., 2005; Stefan van Drongelen et al., 2005). Ser físicamente activo, presenta beneficios en fuerza, autonomía en las actividades diarias y bien estar psicológico en personas con discapacidad física (Hicks et al., 2003; Tordi et al., 2001). Sin embargo, $52,5 \%$ de la actividad diaria es leve y solo $1 \%$ de actividad intensa (Warms, Whitney, y Belza, 2008).

PALABRAS CLAVE: Lesión medular, actividad física, discapacidad, dolor de hombro, calidad de vida. 


\section{INTRODUCCIÓN}

La lesión medular (LM) es resultado de una enfermedad/tumor (20\%) o trauma $(80 \%)$ (Maestro, 2010) alterando el normal funcionamiento de la medula y con pérdida de sensibilidad, acción motorice, visceral y alteraciones sexuales y tróficas. Las consecuencias resultantes dependen del nivel y grado de lesión, la sección de medular afectada y el tempo desde la lesión; se la lesión es debajo de T1 se designa de paraplejia y arriba por tetraplegia (Lianza, Casalis, y Greve, 2007; Price, 2010).

El objetivo de este estudio es de realizar una revisión bibliográfica sobre la relación entre calidad de vida, prevalencia de dolor de hombro y nivel de actividad física en personas con lesión medular.

\section{Lesiones por uso de silla de ruedas manual}

El uso de silla de ruedas manual en sujetos con lesión medular requiere una carga continua sobre la extremidad superior en desplazamiento, transferencias, elevaciones y coger objetos (S van Drongelen, van der Woude, y Veeger, 2011; Stefan Van Drongelen et al., 2005; Stefan van Drongelen et al., 2005). Este tipo de quejas es frecuente a nivel del hombro (30\% a 73\%) (Boninger et al., 2003) y del síndrome de canal carpiano (49\% a 73\%) con la progresión de años desde la lesión (Boninger et al., 2005).

Las lesiones resultantes del uso excesivo de silla de ruedas manual se deben a factores individuales como la capacidad física, ambientales como inclinaciones $u$ escalones y la magnitud y frecuencia de carga aplicada (Hastings y Goldstein, 2004).

\section{Dolor de hombro}

Los casos de dolor de hombro son observados en $78 \%$ de sujetos con tetraplegia y $59 \%$ de paraplejia desde que comienzan a utilizar silla de ruedas manual (Curtis et al., 1999). Cerca del mismo porcentaje (60\%) de dolor de hombro ha sido observado por jugadores de baloncesto en silla de ruedas cuando comparados con no parapléjicos $(51,17 \%)$ (Yildirim, Comert, y Ozengin, 2010). Los sujetos con reportan más dolor en actividades como levantar la silla de ruedas manual para dentro del coche, subir rampas $u$ inclinaciones y realizar actividades de vida diaria (Samuelsson, Tropp, y Gerdle, 2004).

\section{Actividad Física}

Ser físicamente activo presenta beneficios en fuerza, autonomía en las actividades de vida diaria y bien estar psicológico en personas con incapacidad física (Hicks et al., 2003; Tordi et al., 2001). Pero, eso depende factores inmutables (edad o genero) y factores modificables (determinantes psicosociales) (Humpel, Owen, y Leslie, 2002). Los segundos, son importantes en el contexto de promoción de actividad física porque pueden ser estimulados en las actividades (Ploeg, Beek, y Woude, 2004) y pueden presentar beneficios a nivel de salud y condición física (Barata, 1997).

El 55.5\% de los sujetos con lesión medular reportan estar haciendo actividad física y que una gran mayoría $(83,2 \%)$ hacen ejercicios recreativos y deporte. Y se observa que los tetrapléjicos $(38,6 \%)$ son menos activos que los parapléjicos $(54,8 \%)$ (Anneken, Hanssen-Doose, Hirschfeld, Scheuer, y Thietje, 2010). En general, en los 
sujetos con lesión medular, los hombres son más activos que las mujeres y los usuarios de silla de ruedas manual son más activos que los usuarios de sillas de ruedas eléctricas (Ginis et al., 2010). Sin embargo, 52,5\% de la actividad diaria es en actividad leve y solo $1 \%$ de actividad intensa (Warms, Whitney, y Belza, 2008) durante el uso de sillas de ruedas manuales.

Un factor que puede aclarar este hecho es la cercanía de la casa de los sujetos con las instalaciones deportivas (Arbour y Martin Ginis, 2009; Diez Roux et al., 2007; Reed y Phillips, 2005), porque promociona hábitos de actividad física. Pero, la presencia de barreras a nivel personal, de recursos $u$ arquitectura pueden limitar la actividad física de estas personas y el desplazamiento a las instalaciones deportivas, siendo muy importante comprender la interacción compleja de estos variables para el desarrollo de estrategias y promoción de un estilo de vida saludable en personas con discapacidad (Scelza, Kalpakjian, Zemper, y Tate, 2005; Sisto y Evans, 2014).

\section{Calidad de vida}

La calidad de vida se presenta como un factor bastante importante y que influye bastante en las actividades de personas con lesión medular. Los sujetos con lesión medular presentan valores más bajos de calidad de vida que las personas sin discapacidad, con elevado impacto en la función, limitaciones físicas, dolor, función social y vialidad. Y el nivel neurológico presenta una gran influencia en la calidad de vida, especialmente en tetraplegia (Middleton, Tran, y Craig, 2007).

La participación en actividades y los factores personales demuestran ser factores significantes en la calidad de vida de sujetos con lesión medular. El nivel de participación influye en la satisfacción y bien - estar, por lo tanto, sujetos con mas independencia en actividades presentan valores más altos de calidad de vida (Chang, Wang, Jang, y Wang, 2012).

A nivel social, se observa que los sujetos con lesión medular que indican una mayor diferencia entre dedicación y valorización de trabajo indican valores menores de calidad de vida, menor satisfacción con la salud, actividades de vida diaria, relacionamientos y condiciones de vida (Fekete, Wahrendorf, Reinhardt, Post, y Siegrist, 2014).

La depresión en lesión medular es relacionada con las habilidades sociales y esto influye en la calidad de vida (Müller et al., 2015). Sin embargo, se observa que sujetos lesión medular con depresión consideran la práctica de ejercicio y antidepresivos como las opciones más favorables. Y en sujetos sin depresión, el ejercicio es una de las primeras elecciones (Fann et al., 2013), además, la satisfacción y calidad de vida mejoran en sujetos que desarrollan actividades físicas (Bassett y Martin Ginis, 2009).

Otra gran influencia en la calidad de vida en sujetos con lesión medular son las ulceras de presión. $65.3 \%$ reportan que las ulceras condicionan su actividad al paso que $12 \%$ reportan su calidad de vida como mala o muy mala en resultado de este problema. Y en la presencia de tres ulceras, los sujetos reportan una calidad de vida significativamente más baja con $52 \%$ indicando la calidad de vida como mala o muy mala. Y el impacto principal en las actividades diarias es en personas con tetraplegia principalmente en la capacidad de cuidarse, alimentarse y desplazarse (Lala, Dumont, Leblond, Houghton, y Noreau, 2014). 
Otro factor bastante importante es el tiempo desde la lesión, porque se observa una relación negativa entre la calidad de vida y los años desde la lesión medular. Los sujetos con mas tempo desde la lesión medular, presenta peores valores de calidad de vida en los dominios físico y psicológico, pero a nivel psicológico este factor influye menos en sujetos con mayor grado académico (Saadat et al., 2010).

\section{CONCLUSIONES}

Se observa que la calidad de vida en personas con lesión medular disminuí al pasar el tiempo de la lesión y aumenta la prevalencia de dolor de hombro con el uso continuado de silla de ruedas manual. Estos factores son bastante importantes para las personas con lesión medular porque van influir en la capacidad de realizar actividad física regular o tener una integración social y bien estar psicológico.

La actividad física se presenta como un factor potenciador de una mejora de la fuerza física y capacidad de desplazamiento de la silla de ruedas, ayudando a disminuir las barreras físicas y como un factor de inclusión, participación social y de mejoría del bien - estar de los sujetos con lesión medular.

Para que se promueva la inclusión es necesario promover un mejor acceso a instalaciones deportivas, reducir las barreras para incentivar a que las personas con lesión medular practiquen más deporte y de entre ellos que se promuevan las estrategias para mejor inclusión de las mujeres en actividades físicas adaptadas.

\section{BIBLIOGRAFÍA}

Anneken, V., Hanssen-Doose, a, Hirschfeld, S., Scheuer, T., y Thietje, R. (2010). Influence of physical exercise on quality of life in individuals with spinal cord injury. Spinal Cord, 48(5), 393-9. doi:10.1038/sc.2009.137

Arbour, K. P., y Martin Ginis, K. a. (2009). The relationship between physical activity facility proximity and leisure-time physical activity in persons with spinal cord injury. Disability and Health Journal, 2(3), 128-35. doi:10.1016/j.dhjo.2009.01.003

Barata, T. (1997). O consumo de energia. In T. Barata y et al (Eds.), Actividade Física e Medicina Moderna (1st ed., pp. 49 - 55). Odivelas: Europress.

Bassett, R. L., y Martin Ginis, K. a. (2009). More than looking good: impact on quality of life moderates the relationship between functional body image and physical activity in men with SCI. Spinal Cord: The Official Journal of the International Medical Society of Paraplegia, 47(3), 252-256. doi:10.1038/sc.2008.114

Boninger, M. L., Dicianno, B. E., Cooper, R. a, Towers, J. D., Koontz, A. M., y Souza, A. L. (2003). Shoulder magnetic resonance imaging abnormalities, wheelchair propulsion, and gender. Archives of Physical Medicine and Rehabilitation, 84(11), 1615-1620. doi:10.1053/S0003-9993(03)00282-X

Boninger, M. L., Koontz, A. M., Sisto, S. A., Dyson-Hudson, T. a., Chang, M., Price, R., y Cooper, R. a. (2005). Pushrim biomechanics and injury prevention in spinal cord injury: Recommendations based on CULP-SCI investigations. The Journal of Rehabilitation Research and Development, 42(3sup1), 9 - 20. doi:10.1682/JRRD.2004.08.0103 
Chang, F.-H., Wang, Y.-H., Jang, Y., y Wang, C.-W. (2012). Factors associated with quality of life among people with spinal cord injury: application of the International Classification of Functioning, Disability and Health model. Archives of Physical Medicine and Rehabilitation, 93(12), 2264-70. doi:10.1016/j.apmr.2012.06.008

Curtis, K. a, Drysdale, G. a, Lanza, R. D., Kolber, M., Vitolo, R. S., y West, R. (1999). Shoulder pain in wheelchair users with tetraplegia and paraplegia. Archives of Physical Medicine and Rehabilitation, 80(4), 453-7. Retrieved from http://www.ncbi.nlm.nih.gov/pubmed/10206610

Diez Roux, A. V, Evenson, K. R., McGinn, A. P., Brown, D. G., Moore, L., Brines, S., y Jacobs, D. R. (2007). Availability of recreational resources and physical activity in adults. American Journal of Public Health, 97(3), 493-9. doi:10.2105/AJPH.2006.087734

Fann, J. R., Crane, D. a., Graves, D. E., Kalpakjian, C. Z., Tate, D. G., y Bombardier, C. H. (2013). Depression treatment preferences after acute traumatic spinal cord injury. Archives of Physical Medicine and Rehabilitation, 94(12), 2389-2395. doi:10.1016/j.apmr.2013.07.004

Fekete, C., Wahrendorf, M., Reinhardt, J. D., Post, M. W. M., y Siegrist, J. (2014). Work stress and quality of life in persons with disabilities from four European countries: the case of spinal cord injury. Quality of Life Research : An International Journal of Quality of Life Aspects of Treatment, Care and Rehabilitation, 23(5), 1661-71. doi:10.1007/s11136-013-0610-7

Ginis, K. a M., Latimer, A. E., Arbour-Nicitopoulos, K. P., Buchholz, A. C., Bray, S. R., Craven, B. C., ... Wolfe, D. L. (2010). Leisure time physical activity in a population-based sample of people with spinal cord injury part I: demographic and injury-related correlates. Archives of Physical Medicine and Rehabilitation, 91(5), 722-8. doi:10.1016/j.apmr.2009.12.027

Hastings, J., y Goldstein, B. (2004). Paraplegia and the shoulder. Phys Med Rehabil Clin N Am, 15, 699 - 718.

Hicks, a L., Martin, K. a, Ditor, D. S., Latimer, a E., Craven, C., Bugaresti, J., y McCartney, N. (2003). Long-term exercise training in persons with spinal cord injury: effects on strength, arm ergometry performance and psychological wellbeing. Spinal Cord, 41(1), 34-43. doi:10.1038/sj.sc.3101389

Humpel, N., Owen, N., y Leslie, E. (2002). Environmental factors associated with adults' participation in physical activity: a review. American Journal of Preventive Medicine, 22(3), 188-99. Retrieved from http://www.ncbi.nlm.nih.gov/pubmed/11897464

Lala, D., Dumont, F. S., Leblond, J., Houghton, P. E., y Noreau, L. (2014). The Impact of Pressure Ulcers on Individuals Living with a Spinal Cord Injury. Archives of Physical Medicine and Rehabilitation, 2312-2319. doi:10.1016/j.apmr.2014.08.003

Lianza, S., Casalis, M. E. P., y Greve, J. M. D. (2007). A Lesão Medular. In S. Lianza (Ed.), Medicina de Reabilitação (4th ed., pp. 322 - 323). Rio de Janeiro: Guanabara Koogan. 
Maestro, M. A. (2010). Conceptos generales sobre el síndrome de lesíon medular. In A. Esclarín de Ruz (Ed.), Lesión Medular: Enfoque multidisciplinario (p. 332). Madrid: Medica Panamericana.

Middleton, J., Tran, Y., y Craig, A. (2007). Relationship between quality of life and selfefficacy in persons with spinal cord injuries. Archives of Physical Medicine and Rehabilitation, 88(12), 1643-8. doi:10.1016/j.apmr.2007.09.001

Müller, R., Peter, C., Cieza, A., Post, M. W., Van Leeuwen, C. M., Werner, C. S., y Geyh, S. (2015). Social skills: a resource for more social support, lower depression levels, higher quality of life, and participation in individuals with spinal cord injury? Archives of Physical Medicine and Rehabilitation, 96(3), 447-55. doi:10.1016/j.apmr.2014.09.006

Ploeg, H. P. Van Der, Beek, A. J. Van Der, y Woude, L. H. V. Van Der. (2004). Physical Activity for People with, 34(10), 639-649.

Price, M. (2010). Energy expenditure and metabolism during exercise in persons with a spinal cord injury. Sports Medicine (Auckland, N.Z.), 40(8), 681-96. doi:10.2165/11531960-000000000-00000

Reed, J. A., y Phillips, D. A. (2005). and the Proximity of Exercise Facilities Undergraduate University Students. Journal of American College Health, 53(6).

Saadat, S., Javadi, M., Divshali, B. S., Tavakoli, A. H., Ghodsi, S. M., Montazeri, A., y Rahimi-Movaghar, V. (2010). Health-related quality of life among individuals with long-standing spinal cord injury: a comparative study of veterans and nonveterans. BMC Public Health, 10, 6. doi:10.1186/1471-2458-10-6

Samuelsson, K. a M., Tropp, H., y Gerdle, B. (2004). Shoulder pain and its consequences in paraplegic spinal cord-injured, wheelchair users. Spinal Cord, 42(1), 41-6. doi:10.1038/sj.sc.3101490

Scelza, W. M., Kalpakjian, C. Z., Zemper, E. D., y Tate, D. G. (2005). Perceived Barriers to Exercise in People with Spinal Cord Injury. American Journal of Physical Medicine y Rehabilitation, 84(8), 576-583. doi:10.1097/01.phm.0000171172.96290.67

Sisto, S. A., y Evans, N. (2014). Activity and Fitness in Spinal Cord Injury: Review and Update. Current Physical Medicine and Rehabilitation Reports, 2(3), 147-157. doi:10.1007/s40141-014-0057-y

Tordi, N., Dugue, B., Klupzinski, D., Rasseneur, L., Rouillon, J. D., y Lonsdorfer, J. (2001). Interval training program on a wheelchair ergometer for paraplegic subjects, 532-537.

van Drongelen, S., van der Woude, L. H., Janssen, T. W., Angenot, E. L., Chadwick, E. K., y Veeger, D. H. (2005). Glenohumeral Contact Forces and Muscle Forces Evaluated in Wheelchair-Related Activities of Daily Living in Able-Bodied Subjects Versus Subjects With Paraplegia and Tetraplegia. Archives of Physical Medicine and Rehabilitation, 86(7), 1434-1440. doi:10.1016/j.apmr.2005.03.014

Van Drongelen, S., Van der Woude, L. H., Janssen, T. W., Angenot, E. L., Chadwick, E. K., y Veeger, D. H. (2005). Mechanical load on the upper extremity during 
wheelchair activities. Archives of Physical Medicine and Rehabilitation, 86(6), 1214-20. doi:10.1016/j.apmr.2004.09.023

van Drongelen, S., van der Woude, L. H. V, y Veeger, H. E. J. (2011). Load on the shoulder complex during wheelchair propulsion and weight relief lifting. Clinical Biomechanics (Bristol, Avon), 26(5), 452-7. doi:10.1016/j.clinbiomech.2011.01.006

Warms, C. a, Whitney, J. D., y Belza, B. (2008). Measurement and description of physical activity in adult manual wheelchair users. Disability and Health Journal, 1(4), 236-44. doi:10.1016/j.dhjo.2008.07.002

Yildirim, N. U., Comert, E., y Ozengin, N. (2010). Shoulder pain: a comparison of wheelchair basketball players with trunk control and without trunk control. Journal of Back and Musculoskeletal Rehabilitation, 23(2), 55-61. doi:10.3233/BMR-20100250 